\title{
Specialized Membrane Domains for Water Transport in Glial Cells: High-Resolution Immunogold Cytochemistry of Aquaporin-4 in Rat Brain
}

\author{
Søren Nielsen, ${ }^{1 \mathrm{a}}$ Erlend Arnulf Nagelhus, ${ }^{2 a}$ Mahmood Amiry-Moghaddam, ${ }^{2}$ Charles Bourque, ${ }^{3}$ Peter Agre, ${ }^{4}$ \\ and Ole Petter Ottersen ${ }^{2}$ \\ ${ }^{1}$ Department of Cell Biology, Institute of Anatomy, University of Aarhus, 8000 Aarhus, Denmark, ${ }^{2}$ Department of Anatomy, \\ Institute of Basic Medical Sciences, University of Oslo, 0317 Oslo, Norway, ${ }^{3}$ Centre for Research in Neuroscience, \\ Montréal General Hospital and McGill University, Montréal, Québec, Canada H3G 1A4, and 4Departments of Biological \\ Chemistry and Medicine, The Johns Hopkins University School of Medicine, Baltimore, Maryland 21205
}

\begin{abstract}
Membrane water transport is critically involved in brain volume homeostasis and in the pathogenesis of brain edema. The cDNA encoding aquaporin-4 (AQP4) water channel protein was recently isolated from rat brain. We used immunocytochemistry and high-resolution immunogold electron microscopy to identify the cells and membrane domains that mediate water flux through AQP4. The AQP4 protein is abundant in glial cells bordering the subarachnoidal space, ventricles, and blood vessels. AQP4 is also abundant in osmosensory areas, including the supraoptic nucleus and subfornical organ. Immunogold analysis demonstrated that AQP4 is restricted to glial mem-
\end{abstract}

branes and to subpopulations of ependymal cells. AQP4 is particularly strongly expressed in glial membranes that are in direct contact with capillaries and pia. The highly polarized AQP4 expression indicates that these cells are equipped with specific membrane domains that are specialized for water transport, thereby mediating the flow of water between glial cells and the cavities filled with CSF and the intravascular space.

Key words: aquaporin-4 water channel; brain water permeability; glial cells; ependymal cells; immunogold electron microscopy; CSF
Water metabolism is of major importance in a number of physiological processes in the CNS including CSF production and absorption, fluid transport across neuropil and vascular endothelium, and cell volume regulation (Fitzsimons, 1992; Robertson, 1992). In addition, water transport may serve to compensate for local changes in osmolality associated with potassium siphoning, which is essential for synaptic transmission. Alterations in water distribution in brain and CSF compartments is a common occurence in multiple neuropathological conditions including brain edema, brain tumors, stroke, hyponatremia, head injuries, and hydrocephalus. Despite its importance, little is known about the cellular and molecular mechanisms involved in transmembrane water movements in brain.

Discovery of aquaporin-1 (Preston et al., 1992) answered the long-standing biophysical question of how water crosses plasma membranes (for review, see Agre et al., 1993; Knepper, 1994). Characterization of aquaporins provided molecular insight into fundamental processes of normal water balance and disorders of

\footnotetext{
Received Aug. 16, 1996; revised Oct. 1, 1996; accepted Oct. 10, 1996.
}

This work was supported by The Novo Nordisk Foundation, the Karen Elise Jensen Foundation, The Danish Medical Research Council, the University of Aarhus Research Foundation, the Biomembrane Research Center at University of Aarhus, National Institutes of Health (Grants HL48268, HL33991, and EY11234), the Norwegian Research Council, and the European Union Biomed Programme (BMH4-CT96-0851). We thank Bjørg Riber, Karen Marie Gujord, Gunnar Lothe, Carina Knudsen, T. Nordby, Trine Møller, Mette Vistisen, and Hanne Weiling for excellent technical assistance. The antibody to GLAST was kindly donated by Dr. N. C. Danbolt (University of Oslo).

Correspondence should be addressed to Peter Agre, The Johns Hopkins University School of Medicine, Department of Biological Chemistry, 725 North Wolfe Street, Baltimore, MD 21205.

${ }^{\text {aS }} \varnothing$ ren Nielsen and Erlend Arnulf Nagelhus contributed equally to this work.

Copyright (C) 1996 Society for Neuroscience $0270-6474 / 96 / 170171-10 \$ 05.00 / 0$ water balance outside brain (for review, see King and Agre, 1996; Nielsen et al., 1996). A cDNA for aquaporin-4 (AQP4) water channel protein was isolated recently from rat brain (Hasegawa et al., 1994; Jung et al., 1994), and abundant AQP4 was noted in brain including in cerebellum, hypothalamus, spinal cord, and ependymal cells lining the ventricles (Jung et al., 1994; Frigeri et al., 1995). Nevertheless, the cellular and subcellular distributions of AQP4 in brain remain unknown, and definition of the sites of AQP4 expression will be essential for understanding its physiological and pathophysiological roles.

Immunocytochemistry and high-resolution immunogold electron microscopy were used to define the sites of AQP4 in brain. AQP4 expression is restricted to ependymal cell lining of the ventricles and glial cell membranes, where the most abundant expression is in perivascular glial processes. Heavy expression is also found in highly vascularized areas and areas known to be involved in osmosensation and regulation of body water balance, including the subfornical organ and supraoptic nuclei. AQP4 has a highly polarized organization in the glial membrane with abundant expression in specific membrane domains associated with brain-blood or brain-liquor interfaces, indicating the presence of specific domains for water transport at these sites. Together, these studies implicate AQP4-bearing glial cells as mediators of water metabolism in brain.

\section{MATERIALS AND METHODS}

Animals. Male Wistar rats weighing between 250 and 300 gm (Møllegaard, Ejby, Denmark) were used in this study. The animals were fed and allowed access to water ad libitum.

Antibodies. Rabbits were immunized with keyhole limpet hemocyanin conjugated with synthetic peptides corresponding to amino acids 280-296 (LL182) or 251-269 (LL179) of the region near the C terminus of AQP4 
Anti-AQP4 (LL182)

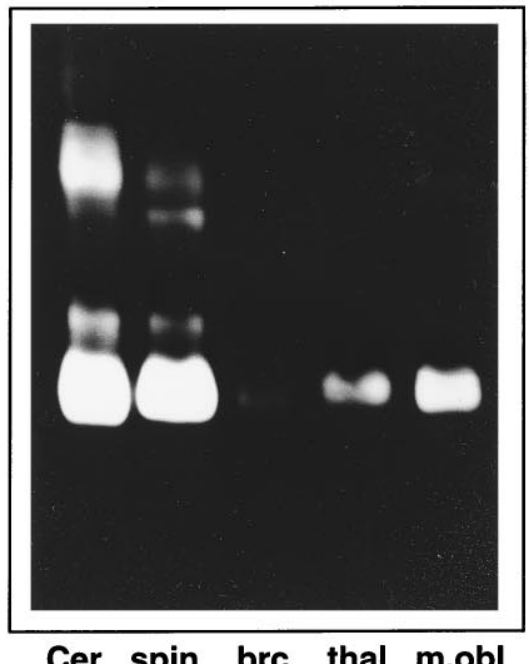

Cer spin brc thal m.obl

\section{Pre-absorption control}

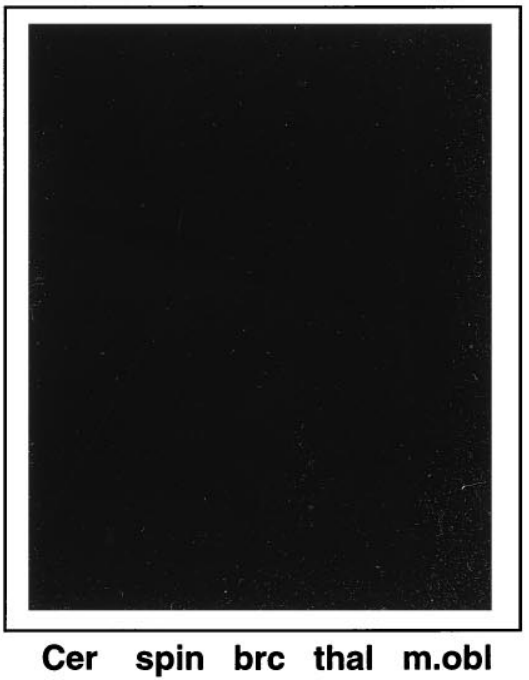

B

B
Anti-AQP4 (LL179)

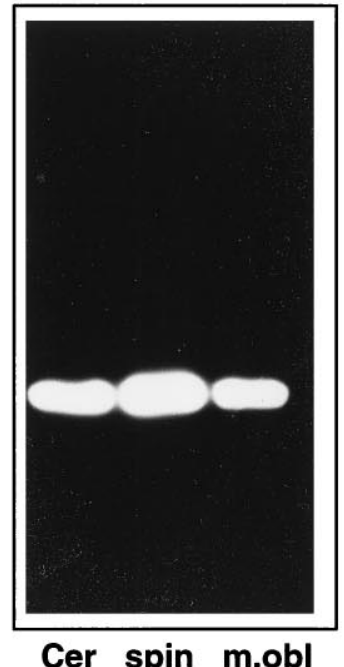

C
Anti-AQP4 (LL182)

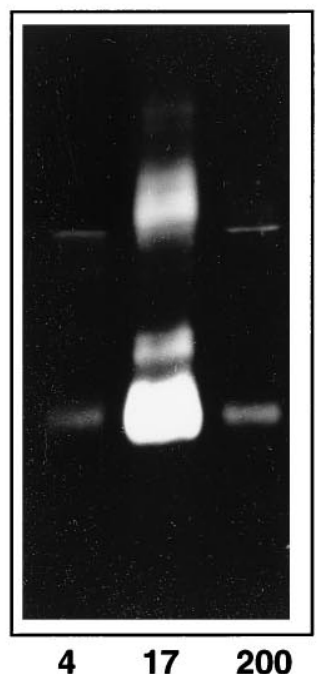

A

Figure 1. AQP4 immunoblots of membrane fractions from rat brain. $A$, Membrane fraction (10 $\mu \mathrm{g} / \mathrm{lane})$ of cerebellum (Cer), spinal cord (spin), cerebral cortex ( $b r c)$, thalamus (thal; and parts of hypothalamus), and medulla oblongata ( $m . o b l)$. The immunoblot was reacted with affinity-purified anti-AQP4 (LL182AP). $B$, Identical to $A$ except that the blot was reacted with affinity-purified antibody previously reacted overnight with immunizing peptide LL182. $C$, Immunoblot of membrane fractions $(10 \mu \mathrm{g} / \mathrm{lane})$ reacted with the second affinity-purified anti-AQP4 antibody (LL179AP). D, Immunoblot of membrane fractions from rat cerebellum using $4000 \times g(4), 17,000 \times g(17)$, and 200,000 $\times g(200)$ membrane pellets. The blot was probed with anti-AQP4 (LL182). Note that longer exposures to radiographic film of LL182 and LL179 blots revealed the presence of higher-molecular-weight bands in all lanes that contained the predominant $\sim 30 \mathrm{kDa}$ band (not shown).

(Jung et al., 1994); the two different antibodies were affinity-purified separately as described previously (Terris et al., 1995). Affinity-purified antibodies to the glutamate transporter GLAST (designated A522) were characterized by Danbolt and colleagues (Chaudhry et al., 1995; Lehre et al., 1995).

Membrane fractionation, electrophoresis, and immunoblotting. Rat CNS was divided into regions and membrane fractions were prepared as described previously (Nielsen et al., 1995b). The membrane samples solubilized in Laemmli buffer were loaded onto 12\% SDS-PAGE gels, and proteins were transferred to nitrocellulose paper by electroelution. Immunoblotting was performed as described previously (Marples et al., 1995; Nielsen et al., 1995b; Terris et al., 1995) with $1.7 \mu \mathrm{g} / \mathrm{ml} \mathrm{IgG}$ (LL182AP) or $2.9 \mu \mathrm{g} / \mathrm{ml} \mathrm{IgG} \mathrm{(LL179AP).}$

Immunocytochemistry. Brains were perfusion fixed with 0.1 or $0.5 \%$ glutaraldehyde and 2 or $4 \%$ paraformaldehyde in 0.1 M sodium cacodylate buffer or $0.1 \mathrm{M}$ phosphate buffer and post-fixed overnight. Immunoperoxidase or immunogold labeling was performed using semithin $(0.9$ $\mu \mathrm{m})$ or ulthrathin $(60-80 \mathrm{~nm})$ cryosections obtained with a ReichertJung cryo-ultramicrotome as described previously (Nielsen et al., 1993; Marples et al., 1995; Terris et al., 1995) using anti-AQP4 (LL182) at 1 $\mu \mathrm{g} / \mathrm{ml} \mathrm{IgG}$.

Using ultrathin sections from cryosubstituted and Lowicryl HM20embedded tissue (van Lookeren Campagne et al., 1991; Chaudhry et al., 1995; Matsubara et al., 1996), we cryoprotected the specimens by immersion in graded concentrations of glycerol $(10,20$, and $30 \%)$ and plunged them rapidly into liquid propane $\left(-170^{\circ} \mathrm{C}\right)$ cooled by liquid nitrogen in a cryofixation unit (KF 80, Reichert, Wien). The samples were immersed in $0.5 \%$ uranyl acetate dissolved in anhydrous methanol $\left(-90^{\circ} \mathrm{C}, 24 \mathrm{hr}\right)$ in a cryosubstitution unit (AFS, Reichert). The temperature was raised in steps of $4^{\circ} \mathrm{C} / \mathrm{hr}$ from -90 to $-45^{\circ} \mathrm{C}$. The samples were washed with anhydrous methanol and infiltrated with Lowicryl HM20 resin at $-45^{\circ} \mathrm{C}$ with a progressive increase in the ratio of resin to methanol. Polymerization was carried out with ultraviolet light $(360$ $\mathrm{nm}$ ) for $48 \mathrm{hr}$. Ultrathin sections were treated with a saturated solution of $\mathrm{NaOH}$ in absolute ethanol $(2-3 \mathrm{sec})$, rinsed, and incubated in the following solutions (at room temperature): (1) $0.1 \%$ sodium borohydride and $50 \mathrm{~mm}$ glycine in Tris-buffered saline containing $0.1 \%$ Triton $\mathrm{X}-100$ (TBST) for $10 \mathrm{~min}$; (2) $2 \%$ human serum albumin (HSA) in TBST (10 min); (3) primary antibodies against AQP4 (1 $\mu \mathrm{g} / \mathrm{ml})$ or
GLAST $(5 \mu \mathrm{g} / \mathrm{ml})$ in TBST containing $2 \%$ HSA for $2 \mathrm{hr}$; (4) $2 \%$ HSA in TBST $(10 \mathrm{~min})$; and (5) gold-conjugated secondary antibody (GAR15, Nanoprobes, Stony Brook, NY) 1:20 in the TBST containing $2 \%$ HSA and polyethyleneglycol $(0.5 \mathrm{mg} / \mathrm{ml}, 2 \mathrm{hr})$.

Double labeling was carried out as described previously (Ottersen et al., 1992) using formaldehyde vapor treatment to avoid interference between the two sequential incubations. AQP4 was visualized by $30 \mathrm{~nm}$ immunogold particles, and GLAST was visualized with $15 \mathrm{~nm}$ particles. Reversal of the antibody sequence did not change the labeling pattern. Preembedding immunogold labeling for electron microscopy was performed by incubating vibratome sections with anti-AQP4 (LL182, $2 \mu \mathrm{g} / \mathrm{ml}$ $\mathrm{IgG})$ followed by goat anti-rabbit Fab fragments coupled to $1.4 \mathrm{~nm}$ gold particles (Nanoprobes). The samples were then subjected to a silver enhancement procedure, treated with $\mathrm{OsO}_{4}$, dehydrated, and embedded in epoxy resin (Durcopan, Fluka). Preembedding immunoperoxidase labeling for light microscopy was performed as described previously (Lehre et al., 1995). The following controls revealed no labeling: (1) affinity-purified antibodies were preincubated with excess immunizing peptide; (2) nonspecific rabbit IgG was substituted for primary antibody; and (3) primary antibody or primary and secondary antibodies were omitted.

\section{RESULTS}

\section{Immunoblot analysis}

Membrane fractions prepared from distinct brain regions contain a major band of $\sim 30 \mathrm{kDa}$ (Fig. 1), the predicted molecular size of AQP4 (Jung et al., 1994). A second band is visible at $\sim 35 \mathrm{kDa}$ and may represent the M1 splice variant, and higher-molecular-weight bands may represent incompletely solubilized oligomers of AQP4 (Lu et al., 1996). Consistent with the overall immunocytochemical labeling patterns (Figs. 2-6), heavy expression is noted in cerebellum and spinal cord with low levels in cerebral cortex (Fig. 1A). Immunolabeling controls using affinity-purified antibody previously reacted with immunizing peptide were negative (Fig. $1 B$ ). The antibodies raised against peptides from two nonoverlapping 

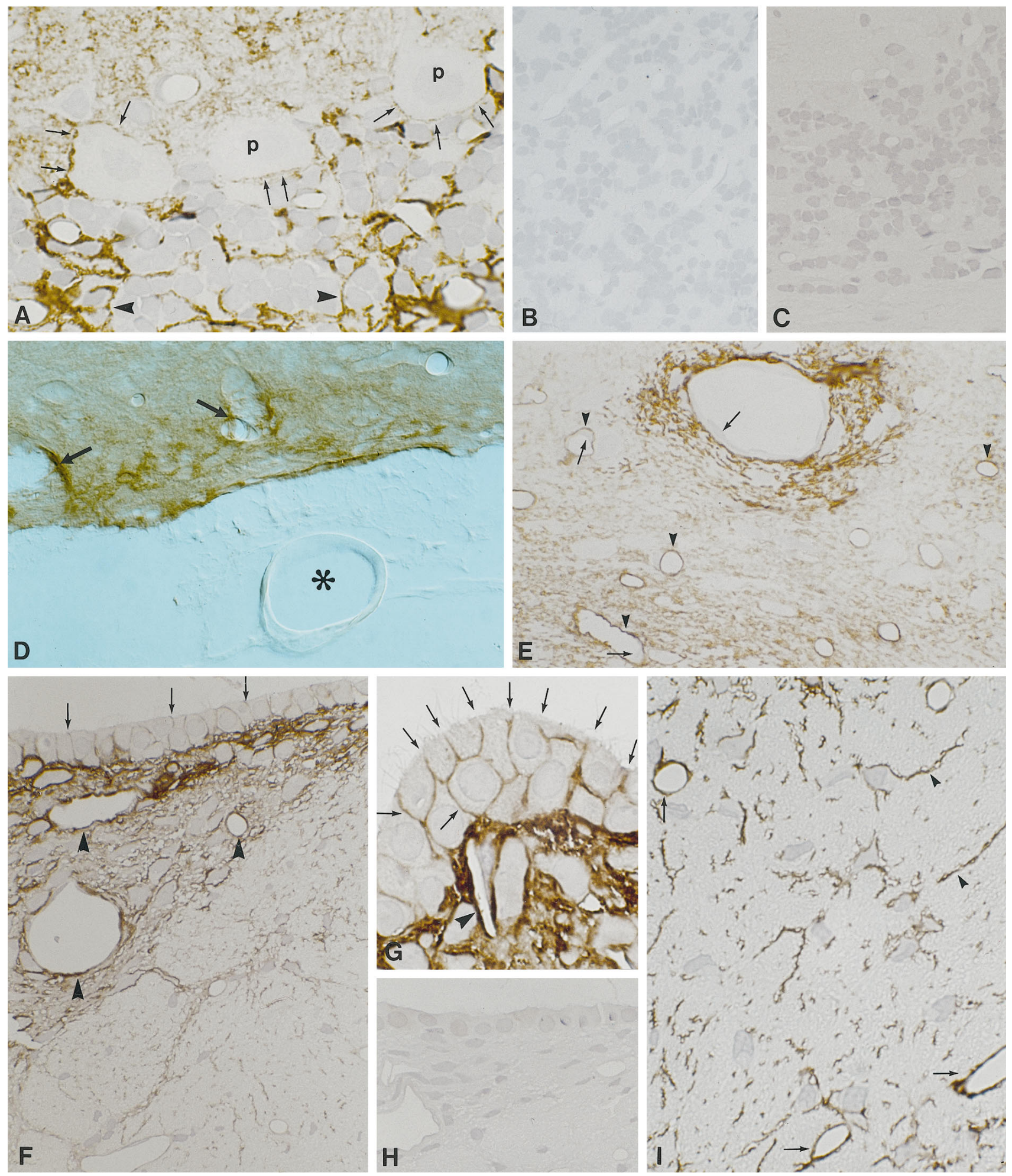

Figure 2. Immunocytochemical localization of AQP4 in rat brain. A, Cryosection of cerebellar cortex. Arrowheads and arrows indicate labeled glial processes in contact with granule cells and Purkinje cells. Magnification, $1000 \times . B$, Control using affinity-purified antibody preabsorbed with excess immunizing peptide reveals no labeling. Magnification, 480×. C, Cryosection of cerebellum incubated with anti-AQP3 (Ecelbarger et al., 1995) demonstrates no labeling. Magnification, $480 \times . D$, Vibratome section of the ventral brain surface at the level of the mesencephalon. Labeling is concentrated close to intracerebral vessels (arrows) and pia but is not associated with vessels in the subarachnoidal space (asterisk) or with arachnoid trabeculae. Magnification, 270×. E, Cryosection of thalamus demonstrates the predominant labeling of glial processes in the vicinity of vessels (arrowheads). Endothelial cells (arrows) and neurons are unlabeled. $F$, Cryosection of subfornical organ. Perivascular glial processes are heavily labeled (arrowheads). Distinct labeling of basolateral plasma membranes of ependymal cells is also present (arrows). Magnification, 480×. G. Higher magnification of basolateral labeling of ependymal cells (arrows) covering the subfornical organ and heavy labeling of perivascular glial cells (arrowhead). Magnification, $1000 \times$. H, Immunolabeling control. Magnification, 480 $\times$. I, Cryosection from spinal cord reveals strong labeling of glial cells (arrowheads) and perivascular glial processes (arrows). Magnification, $480 \times$. 

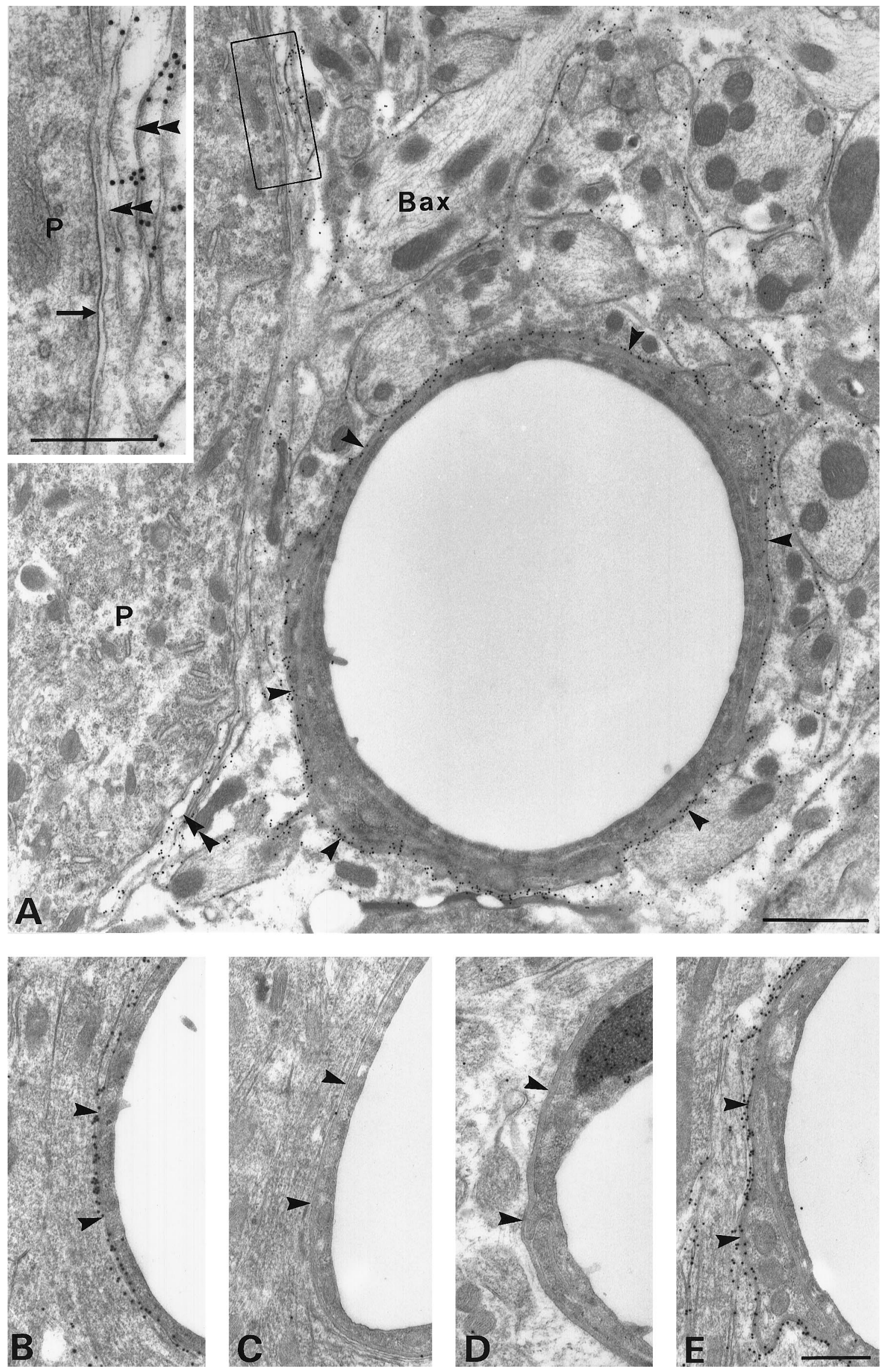
regions of the C-terminal sequence of AQP4 gave similar reactions, confirming the specific labeling of AQP4 (Fig. 1C). Using intracellular vesicle/plasma membrane fractionation methods from kidney (Terris et al., 1995), we determined that AQP4 is most abundant in the plasma membrane-enriched fraction $(17,000 \times g)$ with modest levels in fractions enriched for intracellular vesicles $(200,000 \times g$; Fig. $1 D)$. This distribution is consistent with our immunocytochemical studies (Figs. 3-6).

\section{Selective expression of AQP4 in astrocytes and ependymal cells}

Immunocytochemical preparations showed that AQP4 is restricted to glial cells with morphological features typical of astrocytes (Figs. 2-6) and to subpopulations of ependymal cells (Figs. $2 F, G, 6)$. Glial labeling is found at all levels of the neuraxis, including thalamus (Fig. 2E), mesencephalon (Fig. 2D), cerebellum (Fig. 2A), and spinal cord (Fig. 2I). Glial processes in close vicinity of blood vessels exhibit particularly strong labeling (Figs. $2 D, E, 3 A$ ). Neurons throughout the brain and spinal cord, including Purkinje cells in the cerebellum (Figs. $2 A, 3 A$ ) and magnocellular neurons in the supraoptic nucleus (Fig. 5), were consistently unlabeled. The same was true of the pia and arachnoidea (Fig. 2D), blood vessels (Figs. 2D, 6A), and fibroblasts associated with meninges (Figs. $5 A, 6 A$ ). Controls using antibody previously reacted with the immunizing peptide showed no labeling (Figs. $2 B, H, 3 C$ ). AQP1 is present in the choroid plexus (Nielsen et al., 1993), but immunolabeling of AQP1, AQP2, AQP3, and AQP5 was otherwise negative inside the blood brain barrier of the CNS (Fig. 2C) (data not shown).

\section{Polarized expression of AQP4 in glial cells}

Glial cells exhibit highly differentiated AQP4 immunolabeling with the predominant signal concentrated in glial processes close to or in direct contact with blood vessels (Figs. $2 D, E, 3,5 D$ ), the ependymal layer (Fig. $2 F, G$ ), and pia (Figs. $4 C, 5 C$ ). Individual glial processes show highly polarized expression of AQP4, with a severalfold higher density of gold particles along the membrane domains facing capillaries and pia than along membranes facing the neuropil (Figs. $4 A, D, 5 C, D$ ). This polarity was confirmed with preembedding immunocytochemistry (Fig. $4 B$ ) and with ultrathin cryosections (Fig. 4E). As shown previously (Chaudhry et al., 1995), control experiments showed that the glial glutamate transporter GLAST exhibits the reversed polarity, with the highest immunogold labeling density in membranes abutting the neuropil and surrounding excitatory synapses (Fig. 4D). Preembedding immunogold cytochemistry (Fig. $4 B$ ) and immunogold labeling of ultrathin cryosections (data not shown) demonstrated that AQP4 labeling is restricted to the cytoplasmic aspect of the glial membrane, confirming that the $\mathrm{C}$ terminus of the protein is intracellular (Jung et al., 1994). Different categories of synapses are associated with different levels of AQP4 immunoreactivity. Although weaker than the labeling of glia limitans, the glial processes in contact with parallel fiber synapses on Purkinje cell dendritic spines exhibit clear immunolabeling (Fig. 4C). Glial processes abutting other types of synapses (exemplified in Fig. $6 \mathrm{~A}$, inset) display only modest levels of AQP4 immunolabeling.

\section{Strong non-neuronal expression in osmosensory areas}

AQP4 is heavily expressed in osmosensory areas of brain including hypothalamic magnocellular nuclei (Fig. 5) and the subfornical organ (Figs. $2 F, 6$ ). As in the rest of the brain, the labeling is strictly non-neuronal, with most of the immunoreactivity residing in stacks of glial lamellae (Figs. 5A, 6A). Some AQP4immunopositive lamellae are in direct contact with the unlabeled magnocellular neurons (Fig. 5B); however, these lamellae differ from glial processes in other areas of brain, because they are intensely labeled throughout with little or no polarization of AQP4 distribution. The only exception is at sites of contact with dendrites or axons, where immunolabeling is reduced (Fig. $6 \mathrm{~A}$, inset). Stretches of glial membranes engaged in desmosomes or gap junctions were devoid of labeling (Fig. 6A).

\section{Differentiated expression in ependymal cell membranes}

Moderate densities of gold particles are present in the basolateral membranes of the ependymal cells covering the subfornical organ, whereas the apical membranes are free of labeling (Figs. $2 F, H$, $6 B)$. In contrast, the ependyma of the choroid plexus is devoid of particles along the basolateral as well as the apical surfaces (Fig. $6 C$ ). The labeling intensity of the remaining ependymal covering is intermediate between these two extremes (not shown). All immunolabeling controls confirmed specificity (Fig. 3) (data not shown).

\section{DISCUSSION}

Aquaporins are a family of integral membrane proteins that confer high water permeability to the membranes where they reside (Agre et al., 1993). Aquaporins have been studied most extensively in kidney (Knepper, 1994; Nielsen et al., 1995), but they also exist in other tissues where water transport is important, such as glandular epithelia, anterior chamber of eye, and lung (Nielsen et al., 1993; Frigeri et al., 1995; Raina et al., 1995; King et al., 1996).

Control of water flux and cell volume is of critical importance in the brain, where swelling may be fatal because of the rigid encasement within the cranium. As shown for other tissues, insight into the properties, regulation, and localization of aquaporins in brain may provide a key to understanding how volume homeostasis is normally achieved and how it might be restored if pathologically perturbed. The first member of the aquaporin family, AQP1, was found to be present in the brain, but only in the choroid plexus (Nielsen et al., 1993). AQP4 was identified recently, and initial localization studies (Hasegawa et al., 1994; Jung et al., 1994) suggested that the protein may mediate water flux in the brain at large. Although AQP4 is also found in lung and kidney (Hasegawa et al., 1994; Jung et al., 1994; Frigeri et al., 1995; Terris et al., 1995), its predominant site of expression is in brain.

The physiological role of AQP4 in the brain can only be

\section{$\leftarrow$}

Figure 3. AQP4 in glial processes. Low-magnification electron micrograph showing the distribution of AQP4 immunoreactivity in the Purkinje cell layer $(A)$ and granule cell layer $(B)$ of the cerebellum. Immunogold particles are present along perivascular glial processes (arrowheads) and on glial processes apposed to the Purkinje cell (double arrowheads). The frame in $A$ indicates area enlarged in inset. Immunolabeling is abolished by preadsorption with the peptide used for immunization $(C)$ and by substituting nonimmune $\operatorname{IgG}$ for the primary antibody $(D)$; immunolabeling is not reduced after adsorption with a PKC- $\gamma$ peptide of same length as the immunizing AQP4 peptide $(E)$. Bax, Basket cell axon; $P$, Purkinje cell. Scale bars: $A, 1 \mu \mathrm{m} ; E$, inset in $A, 0.5$ $\mu \mathrm{m} . B-D$ are the same magnification as $E$. 

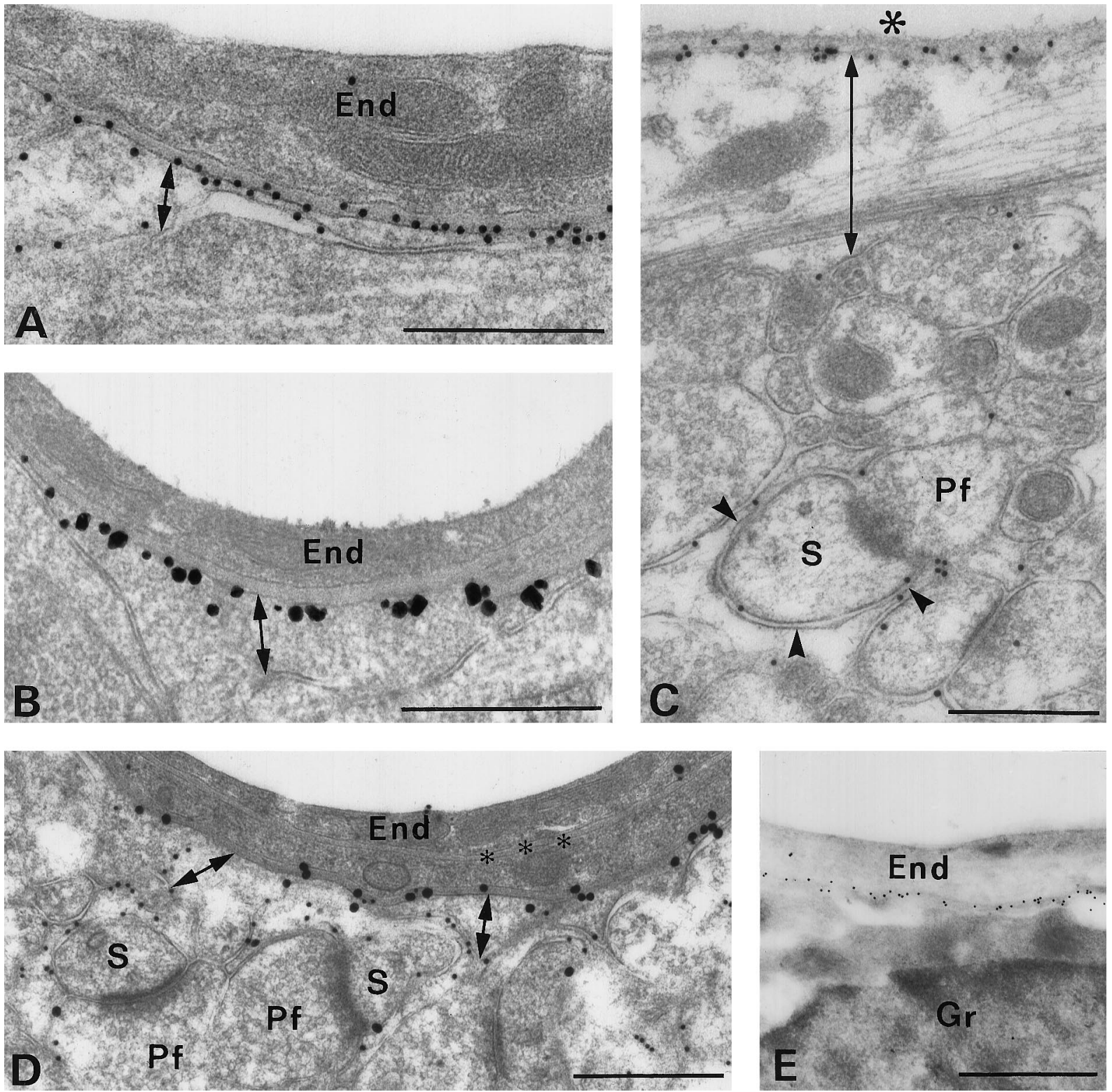

Figure 4. Polarized expression and membrane topology of AQP4 in glial cells. Many immunogold particles are present along glial membranes facing blood vessels $(A, B, D, E)$ and pia $(C)$, but few particles overlie membranes facing the neuropil (double-headed arrows indicate the two membranes). $B$, After preembedding immunogold labeling, silver-intensified immunogold particles localize AQP4 at the cytoplasmic face of the membrane (compare postembedding labeling in $A$ and $D$ ). $D$, Double labeling with antibodies to AQP4 (30 nm gold particles) and the glutamate transporter GLAST (15 nm) reveals distribution of the two antigens to membranes at the opposite poles of the cell. Unlike AQP4, GLAST is concentrated along glial membranes apposed to the neuropil, including those that contact parallel fiber $(P f)$ synapses with Purkinje cell spines $(S)$. E, Postembedding immunogold labeling of cryosection confirms selective labeling of the perivascular glial membrane. End, Endothelium; $G r$, granule cell; $P f$, parallel fiber terminal; $S$, Purkinje cell spines; large asterisk, pial surface; small asterisks, endothelial basal lamina; arrowheads, glial lamellae apposed to parallel fiber synapses. Scale bars: $A-D, 0.5 \mu \mathrm{m} ; E, 1 \mu \mathrm{m}$.

understood after definition of the cellular and subcellular distribution. Because definition of the membrane distribution of AQP4 is still lacking, we have used high-resolution immunogold labeling procedures including cryosubstitution and Lowicryl embedding that had been modified for high sensitivity and optimum preservation of ultrastructure (Matsubara et al., 1996). Standard immunocytochemical procedures with resolution restricted to the cellular level were deemed inadequate, because previous studies of other tissues demonstrated that aquaporins may exhibit a polarized expression that is directly relevant to their physiological roles (Nielsen et al., 1995a; Terris et al., 1995).
A key finding in this study was the highly selective concentration of AQP4 at astrocyte membrane domains facing blood vessels and pia. This implies that the perivascular glial processes and glia limitans may be primary sites of water flux. Lower levels of AQP4 expression occurred in the remainder of the glial cell membrane, with a slight enrichment near certain types of synapse such as the parallel to Purkinje cell synapse in the cerebellum.

A functional polarization of glial cells has also been described in relation to $\mathrm{K}^{+}$flux (Walz, 1989). Investigations of the avascular retina of amphibia provided evidence that excess extracellular $\mathrm{K}^{+}$ resulting from high neuronal activity in the neuropil layers was 

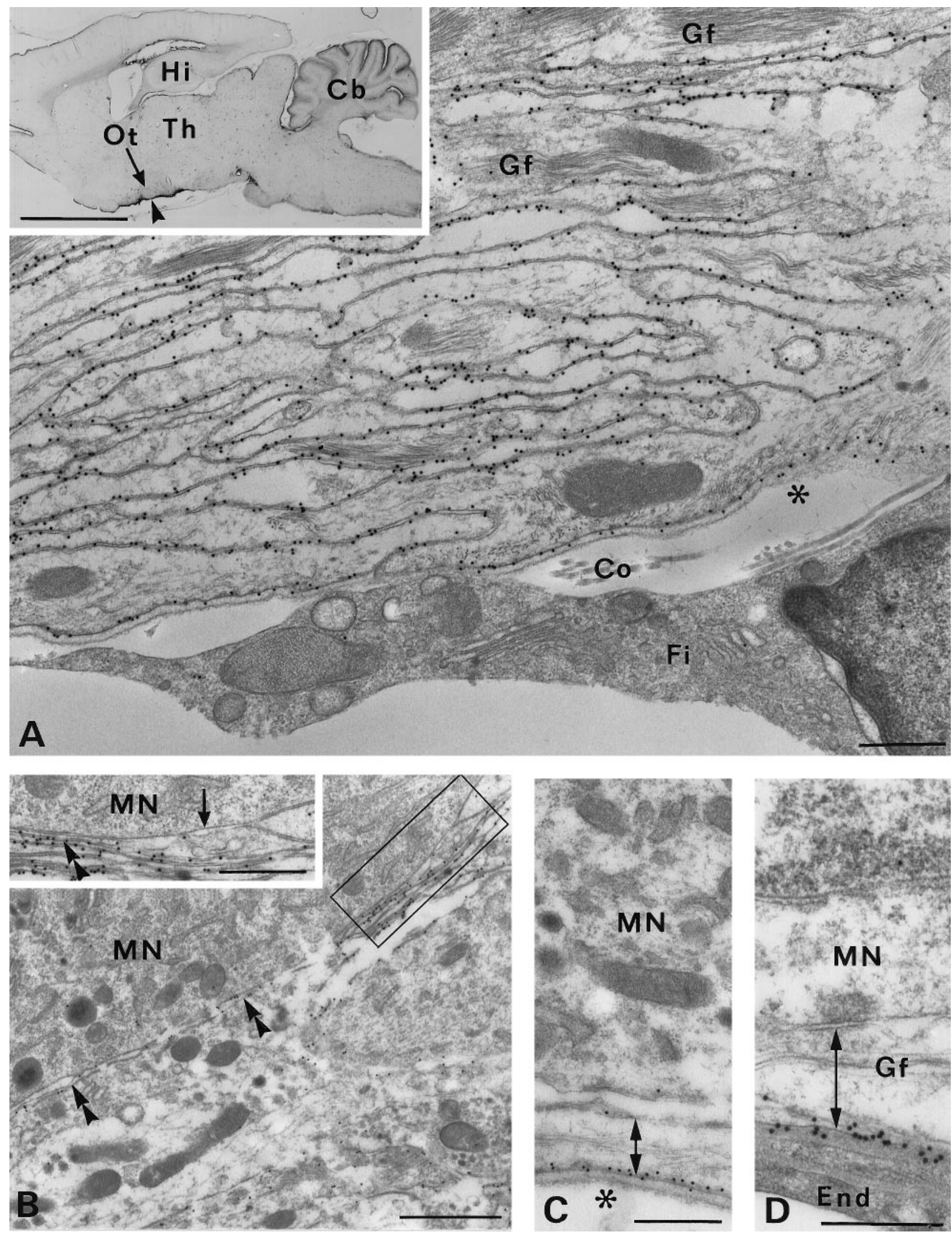

Figure 5. AQP4 in glial lamellae but not in neurons of the supraoptic nucleus. $A$, The ventral glial lamina (arrowhead in inset) associated with the supraoptic nucleus contains numerous glial lamellae that are heavily decorated with immunogold particles. The fibroblast $(F i)$ in the subarachnoidal space (asterisk) is unlabeled. Co, Collagen fibers; $G f$, glial filaments. A, Inset, Sagittal vibratome section $1.9 \mathrm{~mm}$ lateral to midline after light immunolabeling for AQP4. The predominant staining lies along the ventral brain surface corresponding to the position of the supraoptic nucleus (including its retrochiasmatic portion) and in the cerebellum $(C b)$. $H i$, Hippocampus; $O t$, optic tract; $T h$, thalamus. $B-D$, Immunogold particles identify AQP4 in glial membranes associated with pia (double-headed arrow in $C$ ), blood vessels (double-headed arrow in $D$ ), and magnocellular neurons (double arrowheads in $B$ ), but not in neuronal membranes (arrow in inset). End, Endothelial cell; Gf, glial filaments; $M N$, magnocellular neuron; asterisk, pial surface. Frame in $B$ shows area enlarged in inset. Scale bars: $B, 1 \mu \mathrm{m} ; A, C, D$, inset in $B, 0.5 \mu \mathrm{m}$; inset in $A, 5 \mathrm{~mm}$. 

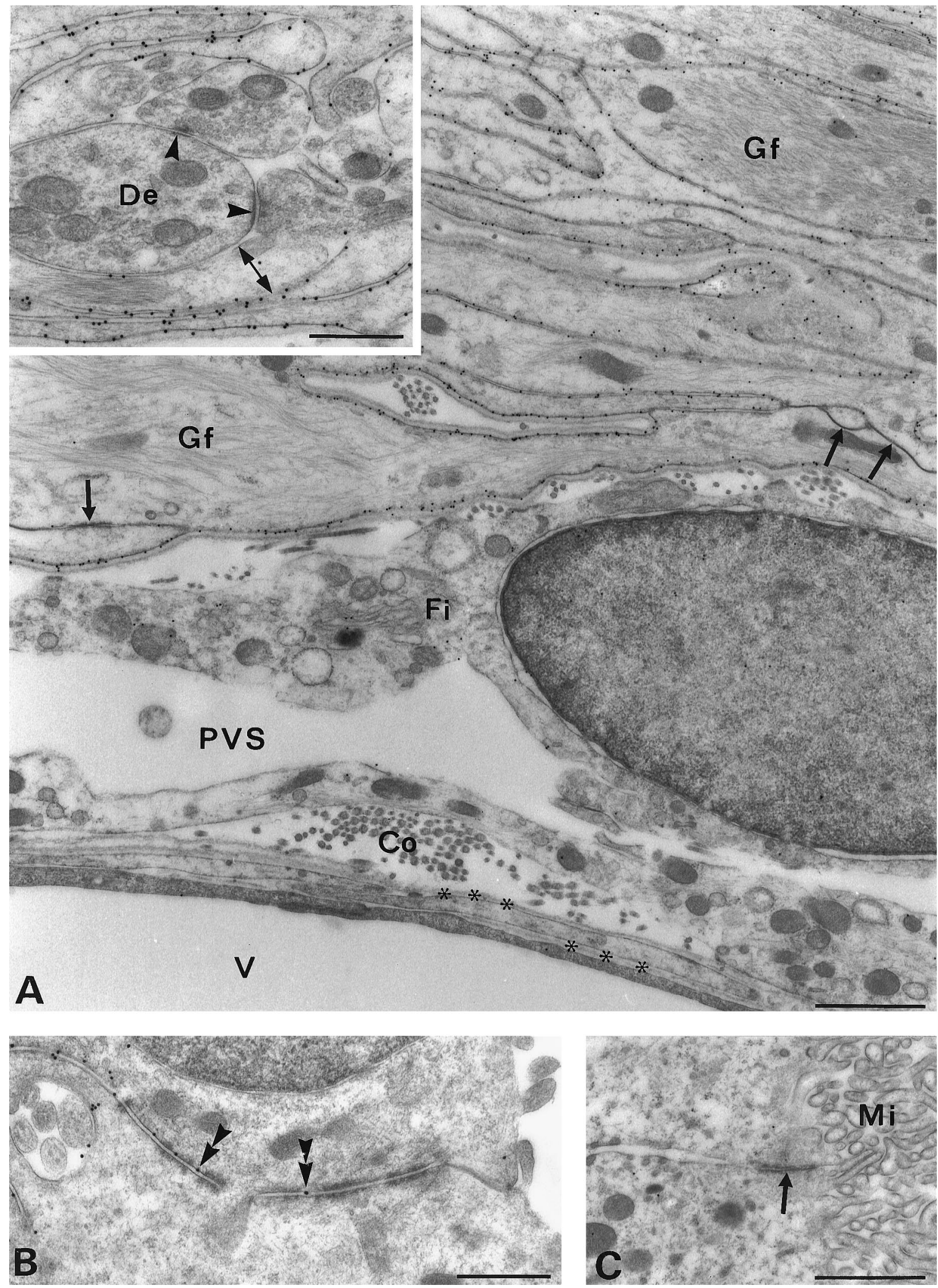

Figure 6. Glial and ependymal expression of AQP4 in the subfornical organ. $A$, Immunogold particles identify AQP4 along the entire glial lamellae except at the membrane domains engaged in gap or adhaerens type junctions (arrows) or contacting neuronal elements (double-headed arrow in inset) The vessel $(V)$ and associated basal laminae (asterisks) are devoid of AQP4 immunolabeling. Co, Collagen; Fi, fibroblast; Gf, glial filaments; PVS, perivascular space. A, Inset, Unlabeled synapses (arrowheads) sandwiched between glial lamellae. The adjacent glial processes are polarized with respect to AQP4 expression (double-headed arrow). De, Dendrite. B, AQP4 labeling of lateral but not apical membranes of ependymal cells covering the subfornical organ (compare Fig. 2). Immunogold particles (double arrowheads) lie between junctional membrane specializations. $C$, Choroid plexus cells and microvilli $(\mathrm{Mi})$ do not express AQP4. Arrow, Apical junctional complex. Scale bars: $A, C, 1 \mu \mathrm{m} ; B$, inset in $A, 0.5 \mu \mathrm{m}$. 
taken up by the Müller glial cells and released through the endfeet into the corpus vitreum (Newman et al., 1984). In agreement, it was estimated that up to $95 \%$ of the cellular $\mathrm{K}^{+}$conductance was concentrated in the endfeet (Newman, 1984). More recent studies showed that such $\mathrm{K}^{+}$siphoning is not unique to the specialized Müller cells but is a property shared by other astrocytes whose perivascular and subpial processes could be considered functionally analogous to the Müller cell endfeet (Newman, 1986; Walz, 1989). The $\mathrm{K}^{+}$flux must be accompanied by a water flux to compensate for changes in osmolarity (Walz, 1989; Sykova, 1992). Thus, an effective $\mathrm{K}^{+}$siphoning (Newman, 1986; Walz, 1989) or $\mathrm{K}^{+}$spatial buffering (Orkand et al., 1966), which is essential for normal brain function, may be dependent on the presence of water channels and may require that the expression of AQP4 be tuned to the $\mathrm{K}^{+}$fluxing capacity. A high concentration of water channels at the perivascular endfeet may also be of importance in brain volume regulation because the endfeet are likely to constitute a primary exit of inorganic as well as organic osmolytes (Nagelhus et al., 1993; Nagelhus et al., 1996).

An efficient transfer of water can only occur at the interfaces with the ventricles and blood vessels if the glial aquaporins are coupled in series with similar molecules in the ependyma or endothelium. Although some AQP4 was found to be expressed by ependymal cells associated with the subfornical organ, AQP4 is weakly expressed in other regions of the ependymal lining and is absent from the choroid epithelium, which is known to contain AQP1 (Nielsen et al., 1993). AQP1 is also present in endothelial cells in a number of organs (Nielsen et al., 1993) but has not been found in brain vessels, as confirmed in this study. Thus, the aquaporin responsible for the high water permeability in brain endothelia remains to be identified.

These results provide strong support for the idea that astrocytes exhibit functionally specialized membrane domains. It was shown previously (Chaudhry et al., 1995) and confirmed here that the glutamate transporter GLAST is more strongly expressed at glial membranes facing the neuropil than at those facing vessels or pia. AQP4 displays an even more pronounced polarization at the opposite membrane, as demonstrated directly in double-labeled preparations. Taken together, these data suggest that the targeting of proteins to the glial cell membrane is precisely regulated. The possibility should be considered that AQP4 is tethered to the extracellular matrix proteins associated with the basal laminae of endothelial cells and pia.

The glial lamellae associated with osmosensory brain regions, including the supraoptic nucleus and subfornical organ, showed a unique distribution of AQP4. At these sites, AQP4 was strongly expressed along the entire glial cell membrane, except at the membrane stretches engaged in intracellular junctions or facing neuronal elements. This suggests that AQP4 serves special functions in these cells. The high concentration of AQP4 would allow the glial lamellae to respond quickly to changes in extracellular osmolarity. This is interesting because the glial lamellae are known to be intimately associated with neuronal elements (Sofroniew et al., 1981; Armstrong et al., 1982), including dendrites and cell bodies of the stretch-sensitive magnocellular neurons (Oliet et al., 1993). It is likely that the glial lamellae, through an interaction with these neurons, could act as a transducer or amplifier in the osmoregulatory response. Although in situ hybridization studies previously suggested the presence of AQP4 in osmosensory neurons and Purkinje cells (Jung et al., 1994), no neurons in the osmosensory areas or in other brain regions displayed AQP4 that was detected by our immunocytochemical studies. Allowing for the possibility that neurons may exhibit an unrecognized aquaporin, this finding suggests that the regulation of water flux and volume control in the brain are functions that depend primarily on glial cells and AQP4.

\section{REFERENCES}

Agre P, Preston GM, Smith BL, Jung JS, Raina S, Moon C, Guggino WB, Nielsen S (1993) Aquaporin CHIP: the archetypal molecular water channel. Am J Physiol 265:F463-F476.

Armstrong WE, Schoeler J, McNeill TH (1982) Immunocytochemical, Golgi and electron microscopic characterization of putative dendrites in the ventral glial lamina of the rat supraoptic nucleus. Neuroscience 7:679-694.

Chaudhry FA, Lehre KP, van Lookeren Campagne M, Ottersen OP, Danbolt NC, Storm-Mathisen J (1995) Glutamate transporters in glial plasma membranes: highly differentiated localizations revealed by quantitative ultrastructural immunocytochemistry. Neuron 15:711-720.

Ecelbarger C, Terris J, Frindt G, Echevarria M, Marples D, Nielsen S, Knepper MA (1995) Aquaporin-3 water channel localization and regulation in rat kidney. Am J Physiol 269:F663-F672.

Fitzsimons JT (1992) Physiology and pathophysiology of thirst and sodium appetite. In: The kidney: physiology and pathophysiology (Seldin DW, Giebisch G, eds), pp 1615-1648. New York: Raven.

Frigeri A, Gropper MA, Umenishi F, Kawashima M, Brown D, Verkman AS (1995) Localization of MIWC and GLIP water channel homologs in neuromuscular epithelial and glandular tissues. J Cell Sci 108:2993-3002.

Hasegawa H, Ma T, Skach W, Matthay MA, Verkman AS (1994) Molecular cloning of a mercurial-insensitive water channel expressed in selected water-transporting tissues. J Biol Chem 269:5497-5500.

Jung JS, Bhat RV, Preston GM, Guggino WB, Baraban JM, Agre P (1994) Molecular characterization of an aquaporin cDNA from brain: candidate osmoreceptor and regulator of water balance. Proc Natl Acad Sci USA 91:13052-13056.

King LS, Agre P (1996) Pathophysiology of the aquaporin water channels. Annu Rev Physiol 58:619-648.

King LS, Nielsen S, Agre P (1996) Aquaporin-1 water channel protein in lung: ontogeny, steroid-induced expression, and distribution in rat. J Clin Invest 97:2183-2191.

Knepper MA (1994) The aquaporin family of molecular water channels. Proc Natl Acad Sci USA 91:6255-6258.

Lehre KP, Levy LM, Ottersen OP, Storm Mathisen J, Danbolt NC (1995) Differential expression of two glial glutamate transporters in the rat brain: quantitative and immunocytochemical observations. J Neurosci 15:1835-1853.

Lu M, Lee MD, Smith BL, Jung JS, Agre P, Verdijk MAJ, Merkx G, Rijss JPL, Deen PM (1996) The human aquaporin-4 gene: definition of locus encoding two water channel polypeptides in brain. Proc Natl Acad Sci USA 93:10908-10912.

Marples D, Christensen S, Christensen EI, Ottosen PD, Nielsen S (1995) Lithium-induced downregulation of aquaporin-2 water channel expression in rat kidney medulla. J Clin Invest 95:1838-1845.

Matsubara A, Laake JH, Davanger S, Usami S, Ottersen OP (1996) Organization of AMPA receptor subunits at a glutamate synapse: a quantitative immunogold analysis of hair cell synapses in the rat organ of corti. J Neurosci 16:4457-4467.

Nagelhus EA, Lehmann A, Ottersen OP (1993) Neuronal-glial exchange of taurine during hypo-osmotic stress: a combined immunocytochemical and biochemical analysis in rat cerebellar cortex. Neuroscience 54:615-631.

Nagelhus EA, Lehmann A, Ottersen OP (1996) Neuronal and glial handling of glutamate and glutamine during hypoosmotic stress: a biochemical and quantitative immunocytochemical analysis using the rat cerebellum as a model. Neuroscience 72:743-755.

Newman EA (1984) Regional specialization of retinal glial cell membrane. Nature 309:155-157.

Newman EA (1986) High potassium conductance in astrocyte endfeet. Science 233:453-454.

Newman EA, Frambach DA, Odette LL (1984) Control of extracellular potassium levels by retinal glial cell $\mathrm{K}^{+}$siphoning. Science 225:1174-1175.

Nielsen S, Agre P (1995) The aquaporin family of water channels in kidney. Kidney Int 48:1057-1068. 
Nielsen S, Smith B, Christensen EI, Knepper MA, Agre P (1993) CHIP28 water channels are localized in constitutively water-permeable segments of the nephron. J Cell Biol 120:371-383.

Nielsen S, Smith BL, Christensen EI, Agre P (1993) Distribution of aquaporin CHIP in secretory and resorptive epithelia and capillary endothelia. Proc Natl Acad Sci USA 90:7275-7279.

Nielsen S, Chou CL, Marples D, Christensen EI, Kishore BK, Knepper MA (1995a) Vasopressin increases water permeability of kidney collecting duct by inducing translocation of aquaporin-CD water channels to plasma membrane. Proc Natl Acad Sci USA 92:1013-1017.

Nielsen S, Marples D, Birn H, Mohtashami M, Dalby NO, Trimble WS, Knepper MA (1995b) Expression of VAMP-2-like protein in kidney collecting duct intracellular vesicles: co-localization with aquaporin-2 water channels. J Clin Invest 96:1834-1844.

Nielsen S, Marples D, Frøkiaer J, Knepper M, Agre P (1996) The aquaporin family of water channels in kidney: an update on physiology and pathophysiology of aquaporin-2. Kidney Int 49:1718-1723.

Oliet SH, Bourque CW (1993) Mechanosensitive channels transduce osmosensitivity in supraoptic neurons. Nature 364:341-343.

Orkand RK, Nicholls JG, Kuffler SW (1966) Effect of nerve impulses on the membrane potential of glial cells in the central nervous system of amphibia. J Neurophysiol 29:788-806.

Ottersen OP, Zhang N, Walberg F (1992) Metabolic compartmentation of glutamate and glutamine: morphological evidence obtained by quantitative immunocytochemistry in rat cerebellum. Neuroscience 46:519-534.
Preston GM, Carroll TP, Guggino WB, Agre P (1992) Appearance of water channels in Xenopus oocytes expressing red cell CHIP28 protein. Science 256:385-387.

Raina S, Preston GM, Guggino WB, Agre P (1995) Molecular cloning and characterization of an aquaporin cDNA from salivary, lacrimal, and respiratory tissues. J Biol Chem 270:1908-1912.

Robertson GL (1992) Regulation of vasopressin secretion. In: The kidney: physiology and pathophysiology (Seldin DW, Giebisch G, eds), pp 1595-1613. New York: Raven.

Sofroniew MW, Glasmann W (1981) Golgi-like immunoperoxidase staining of hypothalamic magnocellular neurons that contain vasopressin, oxytocin or neurophysin in the rat. Neuroscience 6:619-643.

Sykova E (1992) Neuronal-astrocytic interactions (Yu CH, Hertz L, Norenberg MD, Sykova E, Waxman SG, eds). Amsterdam: Elsevier.

Terris J, Ecelbarger C, Marples D, Knepper MA, Nielsen S (1995) Distribution of aquaporin-4 water channel expression within rat kidney. Am J Physiol 269:F775-F785.

van Lookeren Campagne M, Oestreicher AB, van der Krift TP, Gispen WH, Verkleij AJ (1991) Freeze-substitution and Lowicryl HM20 embedding of fixed rat brain: suitability for immunogold ultrastructural localization of neural antigens. J Histochem Cytochem 39:1267-1279.

Walz W (1989) Role of glial cells in the regulation of the brain microenvironment. Prog Neurobiol 33:309-333. 